\title{
The Roles of the Gut Microbiota and Toll-like Receptors in Obesity and Nonalcoholic Fatty Liver Disease
}

\author{
Kouichi Miura*, Mitsuaki Ishioka, Katsunori lijima \\ Department of Gastroenterology, Akita University Graduate School of Medicine, Akita, Japan
}

Obesity is characterized by low-grade chronic inflammation and is closely associated with the cardiovascular diseases, diabetes, and nonalcoholic fatty liver disease. Emerging data demonstrate that the gut microbiota contributes to the development of obesity by regulating the innate immune system, including the Toll-like receptors (TLRs): an altered gut microbiota composition and elevated TLR ligands are observed in obese mice and humans. The changes in the gut microbiota include an increased abundance of Firmicutes phylum and a decreased abundance of Bacteroidetes phylum. The population of beneficial bacteria that function as probiotics is decreased whereas harmful bacteria that can produce lipopolysaccharide, a TLR4 ligand, are increased in the obese state. In addition, the gut permeability is increased in obesity, which allows the delivery of larger amounts of bacterial components to the liver through the portal vein. Immune cells recognize these bacterial components through TLRs and produce diverse cytokines that kill invading pathogens. However, the sustained activation of TLR signaling induces host damage due to chronic exposure to harmful cytokines, which are produced from TLR expressing cells, including monocytes/macrophages. In the obese state, the expression of TLR is increased in several organs, including the adipose tissue and the liver. At the cell level, negative regulators of TLR signaling are suppressed, leading to activation of TLR signaling. These alterations promote inflammation in many organs. Thus, the gut microbiota and TLR signaling are therapeutic targets in patients with obesity and its related diseases.

Key words: Obesity, Nonalcoholic fatty liver disease, Gut microbiota, Toll-like receptor

\author{
Received January 22, 2017 \\ Reviewed February 28, 2017 \\ Accepted March 16, 2017 \\ *Corresponding author \\ Kouichi Miura \\ (iD) \\ http://orcid.org/0000-0001-8036-6544 \\ Department of Gastroenterology, Akita \\ University Graduate School of Medicine, \\ 1-1-1 Hondo, Akita-shi, Akita 010-8543, \\ Japan \\ Tel: +81-18-884-6104 \\ Fax: +81-18-836-2611 \\ E-mail:miura116@doc.med.akita-u.ac.jp
}

\section{INTRODUCTION}

Obesity is characterized by low-grade chronic inflammation. More than 600 million people suffer from obesity and its related diseases in the world, and the pandemic has spread to younger generation including children. ${ }^{1}$ Obesity can cause various life-threatening diseases, including cardiovascular diseases, diabetes, and nonalcoholic fatty liver disease (NAFLD). Although an appropriate nutritional intake and exercise are recommended to prevent excessive body weight, this " 21 st century issues" has not yet been conquered. Thus, new approaches are required to reduce the risk of obesity-related diseases.

The innate immune system is closely associated with the devel- opment of obesity. In the innate immune system, Toll-like receptors (TLRs) are pattern recognition receptors that sense bacterial and viral components. ${ }^{2}$ In addition, nutritional factors such as free fatty acids and host-derived substances have been reported as TLR ligands. ${ }^{3}$ These TLR ligands are elevated in obese subjects and TLR signaling is activated at the cell level, leading to an increased production of proinflammatory cytokines. The gut microbiota has attracted a great deal of attention in relation to the development of obesity and its related diseases because it is the major source of TLR ligands. ${ }^{4}$ Thus, the alteration of the gut microbiota composition impairs the host immune response. Recent technological advances have provided further information on the molecular mecha-

Copyright (C) 2017 Korean Society for the Study of Obesity

(a) This is an Open Access article distributed under the terms of the Creative Commons Attribution Non-Commercial License (http://creativecommons.org/licenses/by-nc/4.0/) which permits unrestricted non-commercial use, distribution, and reproduction in any medium, provided the original work is properly cited. 
nisms of obesity, particular in the field of the gut microbiota and TLR signaling.

Here, we review the role of gut microbiota, the TLRs, and the downstream molecules in the development of obesity and NAFLD.

\section{The composition of gut microbiota in obesity and}

\section{NAFLD}

In the last two decades, the role of gut microbiota has attracted much attention in the development of obesity and NAFLD. Gut microbiota assists in the energy intake by digesting complex carbohydrates. In addition, the gut microbiota contributes to obesity by regulating the innate immune system including TLR signaling. Thus, researchers have extensively investigated the composition of the gut microbiota and tried to identify the specific bacteria that cause obesity and NAFLD (Table 1). The Firmicutes and Bacteroidetes phyla are major components of the gut microbiota, followed by the Proteobacteria and Actinobacteria phyla. In 2005, Ley et al. ${ }^{5}$ reported that an increased Firmicutes/Bacteroidetes (F/B) ratio was associated with obesity in a genetically-modified mouse. Indeed, germ-free mice gained body weight after the transplantation of cecal contents harvested from obese mice. ${ }^{6}$ Subsequently, an increased $\mathrm{F} / \mathrm{B}$ ratio has been revealed in diet-induced obese mice ${ }^{7}$ and patients with obesity. ${ }^{8}$ These data demonstrate that the composition of the gut microbiota is closely associated with the development of obesity. Thereafter, Hildebrandt et al. demonstrated that a high fat (HF) diet increased the F/B ratio in genetically lean mice. ${ }^{7}$ Consistent with their report, we have shown that an increased $\mathrm{F} / \mathrm{B}$ ratio is also observed in mice fed a methionine-choline-deficient (MCD) diet that lose body weight. ${ }^{9}$ These data indicate the $\mathrm{F} / \mathrm{B}$ ratio is affected not only by the obese condition but also by the dietary composition.

The abundance of the Proteobacteria and Actinobacteria phyla were increased in mice on a HF diet. ${ }^{7}$ The Proteobacteria phylum includes lipopolysaccharide (LPS)-producing bacteria, including Escherichia coli and Desulfovibrio vulgaris. On the other hand, the Actinobacteria phylum includes Bifidobacterium, which is used as probiotics. Thus, the balance between "good" and "bad" bacteria is important in the development of obesity.

Because an imbalance of the gut microbiota is likely to induce obesity, various attempts have been made to control the gut microbiota in order to improve obesity. Gut sterilization by antibiotic treatment has been used to find the target bacteria. The administration of ampicillin and neomycin improved HF-diet-induced insulin resistance in mice. ${ }^{10}$ The administration of vancomycin and baci-

Table 1. Altered bacterial population in the gut observed in obese subjects

\begin{tabular}{|c|c|c|c|c|c|c|}
\hline Gram & Phylum Level & Order level & Genus level & Species level & Function & Study \\
\hline \multirow[t]{8}{*}{ Positive } & Firmicutes & Clostridiales & Clostridium & C. phytofermentans & Produce SCFAs and ethanol & Ishioka et al. $(2017)^{9}$ \\
\hline & & & Eubacterium & E. rectale, E. halli & Produce SCFAs & Turnbaugh et al. $(2006)^{25}$ \\
\hline & & & Roseburia & & & \\
\hline & & & Anaerostipes & A. caccae & & \\
\hline & & & Ruminococcus & & & \\
\hline & & Lactobacilales & Lactobacillus & $\begin{array}{l}\text { L. acidophilus, L. Bulgaricus, L. casei, } \\
\text { L. paracasei, L. Plantarum }\end{array}$ & Components of probiotics & $\begin{array}{l}\text { Alisi et al. }(2014)^{17} \\
\text { Okubo et al. }(2013)^{20}\end{array}$ \\
\hline & & & Streptococcus & S. thermophilus & & Velayudham et al. (2009)21 \\
\hline & Actinobacteria & Bifidobacteriales & Bifidobacterium & B. breve, $B$. infantis, $B$. longum & & \\
\hline \multirow[t]{7}{*}{ Negative } & Bacteroidetes & Bacteroidales & Bacteroides & B. thetaiotaomicron & Produce SCFAs & Bäckhed et al. (2004) $)^{13}$ \\
\hline & & & & B. fragilis & $\begin{array}{l}\text { Produce toxin and disrupt the } \\
\text { tight junctions }\end{array}$ & Okubo et al. $(2013)^{20}$ \\
\hline & & & Parabacteoides & P. distasonis, P. goldsteinii & $\begin{array}{l}\text { Exerts anti-inflammatory } \\
\text { effects in the colon }\end{array}$ & Ishioka et al. $(2017)^{9}$ \\
\hline & Proteobacteria & Campylobacterales & Helicobacter & H. pylori, H. hepaticus & Source of LPS & Hildebrandt et al. (2009) \\
\hline & & Enterobacteriales & Escherichia & E. coli & & \\
\hline & & Desulfovibrionales & Desulfovibrio & D. vulgaris & & \\
\hline & Verrucomicrobia & Verrucomicrobiales & Akkermansia & A. muciniphila & Maintain the gut barrier function & $\begin{array}{l}\text { Everard et al. }(2013)^{18} \\
\text { Shin et al. }(2014)^{19}\end{array}$ \\
\hline
\end{tabular}

SCFA, short chain fatty acid; LPS, lipopolysaccharide. 
tracin, which can kill bacteria belonging to Firmicutes and Bacteroidetes phyla, improved insulin resistance by augmenting glucagonlike peptide-1 secretion. ${ }^{11}$ On the other hand, the oral administration of vancomycin led to decreased insulin sensitivity in a human study. ${ }^{12}$ Vancomycin mainly reduces the number of Gram-positive bacteria belonging to the Firmicutes phylum. In contrast, amoxicillin, which also kills Gram-negative bacteria, did not affect insulin sensitivity. These data suggest that the gut microbiota play a complex role in the development of obesity.

\section{The composition of gut microbiota in a HF diet}

HF diets are widely used in experimental models of obesity because HF diets can induce the features of human obesity, including insulin resistance and hepatic steatosis. Although HF diet feeding results in lipid accumulation in the liver, the development of steatohepatitis is uncommon. In mice on a HF diet, the increased abundance of the Firmicutes phylum partially depends on increases in the Lactobacilales order and the Ruminococcus genus. The Ruminococcus genus can ferment indigestible carbohydrates to generate short chain fatty acids (SCFAs), including acetate, propionate, and butyrate. Germ-free mice require higher caloric intake to gain body weight in comparison to conventionally reared mice, probably due to the lack of fermentation in the gut. When the gut microbiota from conventionally reared mice were transplanted into the germ-free mice, they rapidly gained body fat. ${ }^{13}$ Thus, mice on a HF diet can use SCFAs as energy in addition to the high calories from dietary fat.

On the other hand, the abundance of the Lactobacilales order is increased in mice on a HF diet., ${ }^{9,14}$ The Lactobacilales order includes lactate-producing bacteria, which are used for probiotics. In addition, the supplementation of probiotics provides beneficial effects in obesity and NAFLD. ${ }^{15,16} \mathrm{~A}$ high-concentration probiotic mixture VSL\#3 contains eight strains belonging to Bifidobacterium (B) and Lactobacillus (L) genera, including B. breve, B. longum, B. infantis, $L$. acidophilus, L. plantarum, L. paracasei, L. bulgaricus and S. thermophiles. In a human study, the administration of VSL \#3 ameliorated the grade of hepatic steatosis in children with NAFLD through the elevation of glucagon-like peptide- $1 .{ }^{17}$ These data suggest that a HF diet defeats the beneficial effects of the endogenous increase in the Lactobacilales order in mice.

The data from earlier studies suggest the existence of specific bac- teria that cause obesity and NAFLD. Everard et al. ${ }^{18}$ demonstrated that the abundance of the Akkermansia (A) genus belonging to the Verrucomicrobia phylum was decreased in mice on a HF diet. A. muciniphila resides in the mucus layer and represents $3-5 \%$ of the gut microbiota in healthy humans and mice. The abundance of $A$. $m u-$ ciniphila was decreased in a mouse model of HF diet-induced obesity and genetically-modified obese mice while the oral administration of A. muciniphila improved the metabolic profiles, including the fat volume, endotoxemia, insulin resistance, and the inflammation of the adipose tissue (AT), in mice on a HF diet. A. muciniphila is believed to maintain the barrier function of the gut by turning over the mucus layer, in which A. muciniphila both promotes the production of mucin from goblet cells and the degradation of mucin. ${ }^{19}$ In addition, the administration of prebiotics retained the abundance of A. muciniphila and provided beneficial effects on the metabolic profiles. ${ }^{18}$ These data suggest that A. muciniphila has a potential to play important roles in the pathogenesis of obesity.

\section{MCD and choline-deficient amino acid-defined (CDAA) diets}

We herein describe the diets that are used in experimental nonalcoholic steatohepatitis (NASH) research, including MCD and CDAA diets. MCD diets are widely used to investigate molecular mechanisms of NASH because a MCD diet can induce hepatic steatosis as well as inflammation within a short period of time. However, the features of mice on a MCD diet are body weight loss, hypoglycemia, and enhanced insulin signaling, which are the opposite of human NASH. There are a few reports on the composition of gut microbiota affected by MCD diet. Okubo et al. ${ }^{20}$ reported that a $\mathrm{MCD}$ diet reduced the prevalence of lactate-producing bacteria, including Lactobacillus and Bifidobacterium, which are believed to have beneficial effects in the host. Indeed, the oral administration of $\mathrm{Lac}$ tobacillus casei strain protected against MCD diet-induced NASH. Velayudham et al. ${ }^{21}$ reported that the administration of VSL\#3 ameliorated the liver fibrosis in MCD diet-induced steatohepatitis.

CDAA diets induce steatohepatitis, including steatosis, inflammation, liver fibrosis, and obesity, which mimic human NASH. Thus, we have used a CDAA diet as a NASH model. ${ }^{22,23}$ We also compared the CDAA diet to other experimental diets and found that the CDAA diet induced the most severe steatohepatitis among 
the diets we examined. As expected, the composition of the gut microbiota was largely different among experimental diets. ${ }^{9}$ In wild type (WT) mice on the CDAA diet, the abundance of Lactobacillus was decreased whereas the abundance of Clostridium in the Firmicutes phylum was increased. Clostridium is well known to ferment indigestible carbohydrates to generate SCFAs. Although the alteration of the gut microbiota is known to evoke different immune responses, few reports have shown that an altered immune response in the gut affects the severity of NASH. We showed that a CDAA diet increased the expression of interleukin 17 (IL-17) in Paneth cells located in the ileum. As a result, the portal IL-17 levels were elevated in mice on the CDAA diet, in which inflammatory cells infiltrate in the liver. IL-17 is well known to function as a proinflammatory cytokine and chemokine. In fact, IL-17 increased the chemokine expression in hepatic macrophages. ${ }^{9}$

A different phenotype has been reported even when WT mice were fed "MCD" or "CDAA" diets. MCD and CDAA diets are available from several companies that provide different nutritional contents. In addition, modified MCD or CDAA diets are used for experimental models of NASH. The body weight and severity of steatohepatitis are affected by the nutritional contents, including methionine, choline, and $\mathrm{fat}^{24}$; thus, attention should be paid to the nutritional contents of the diets and the data should be carefully interpreted.

\section{TLRs in obesity}

TLRs are pattern recognition receptors that sense viral and bacterial components. The gut microbiota serves as a source of TLR ligands in obese individuals. In addition, fatty acids and nucleic acids are potential TLR ligands. Thus, TLRs are molecular targets of the pathogenesis in obesity. TLRs use myeloid differentiation factor 88 (MyD88) and/or Toll-interleukin 1 receptor domain-containing adapter-inducing interferon- $\beta$ (TRIF) as adaptor proteins, leading to the activation of nuclear factor- $\kappa \mathrm{B}(\mathrm{NF}-\mathrm{\kappa B})$ to produce proinflammatory chemokines and chemokines (Fig. 1). These mediators can induce cellular damage and recruit other immune cells to the target organs. Currently, the roles of TLR2 and TLR4 have been extensively investigated in the development of obesity. In addition, interesting data have been released regarding TLR5 and TLR9. In this review article, we focus on the role of these TLRs and their related molecules.

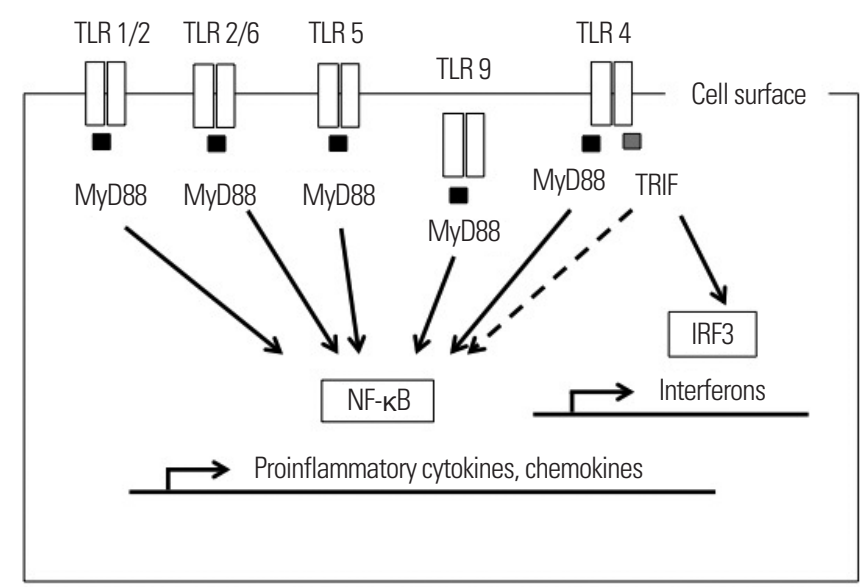

Figure 1. TLR signaling and downstream molecules. Upon TLRs are activated by their ligands, they transmit the signal using adaptor proteins, including MyD88 and/or TRIF. MyD88-depend pathway induces inflammation through NF-кB. On the other hand, TRIF pathway induced genes of interferon regulatory factor (IRF)-3. TLR, toll-like receptor; MyD88, myeloid differentiation factor 88; TRIF, toll-interleukin 1 receptor domain-containing adapter-inducing interferon- $\beta$; IRF3, interferon regulatory factor-3; NF-кB, nuclear factor-кB.

\section{TLR2 and its associated TLRs}

TLR2 recognizes the components of Gram-positive bacteria including peptidoglycan and lipoteichoic acid. Because the abundance of the Firmicutes phylum which includes Gram-positive bacteria is increased in the gut of obese animals and humans ${ }^{8,25}$, circulating TLR2 ligands are presumed to be elevated under obese conditions. In addition, many types of cells, including macrophages and adipocytes, increase the expression of TLR $2 .{ }^{26}$ Leptin, which is elevated under obese conditions, further upregulates the expression of TLR2 in human monocytes and enhances the TLR2 response to bacterial components. ${ }^{27}$ The production of proinflammatory cytokines that is triggered by TLR2 ligands can induce cellular injury and subsequent obesity and insulin resistance. Indeed, TLR2 deficient mice are protected from HF diet-induced obesity. ${ }^{28}$ Thus, TLR2 signaling is considered to promote the progression of obesity.

In line with former studies, we have shown that TLR2 deficient mice were protected from CDAA diet-induced obesity and steatohepatitis. ${ }^{23}$ In that process, the inflammasomes in hepatic macrophages were activated through TLR2. In contrast, Rivera et al. ${ }^{29} \mathrm{re}-$ ported that TLR2 deficient mice were not protected from steatohepatitis when the mice were fed a MCD diet. When mice were fed a MCD diet, they lose body weight and show enhanced insulin sensitivity, opposite to the characteristics of NASH. These findings 
prompted us to examine the composition of the gut microbiota in mice. We found that a MCD diet increased the amount of bacteria belonging to Proteobacteria phylum, which includes LPS-producing bacteria. ${ }^{9}$ In in vitro experiments, a larger amount of ligands is required to activate TLR2 signaling while cells can respond to LPS, a TLR4 ligand, at low concentrations..$^{30}$ Because TLR4 was highly susceptible to LPS in TLR2 deficient mice on a MCD diet, LPS may have activated TLR4 before the activation of TLR2 signaling in these mice.

We also showed that the abundance of Lactobacillus, component of probiotics, was decreased in mice on a MCD diet. ${ }^{9}$ Indeed, the administration of probiotics ameliorated the steatohepatitis and liver fibrosis induced by the MCD diet. ${ }^{20,21}$ Thus, probiotics have the potential to improve obesity and NASH. One question arises as to whether probiotics can be a source of TLR2 ligands because Lactobacillus belongs to the Firmicutes phylum (Table 1). Recently, Zhang et al. ${ }^{31}$ reported a mechanism by which probiotics protect against obesity and NASH through TLR2. They demonstrated that exopolysaccharides (EPS) isolated from Lactobacillus rhamnosus GG ameliorated the obesity and hepatic steatosis induced by a HF diet. Interestingly, EPS also induced the expression of TLR2 but not the expression of other TLRs in the AT and adipocytes. In addition, TLR2 signaling unexpectedly inhibited the lipid accumulation in the adipocytes. Furthermore, the blockade of TLR2 signaling abrogated the EPS-mediated gene expression. These data contrast to those of previous reports, which suggested that TLR2 signaling promotes obesity and NASH. TLR2 is a unique TLR that forms heterodimers with TLR1 or TLR6. In patients with obesity-related NASH, the expression of TLR6 was increased in the peripheral blood mononuclear cells and in the liver. The expression of TLR6 was also correlated with the levels of proinflammatory cytokines. ${ }^{32}$ Indeed, peripheral blood mononuclear cells produced higher amounts of proinflammatory cytokines when the cells were stimulated with a combination of TLR2 and TLR6 agonists. The expression of TLR1 was also increased in the AT and the atherosclerotic aortae of diet-induced obese mice. ${ }^{33}$ These data suggest that a TLR2 heterodimer with TLR6 or TLR1 promotes the inflammatory pathway. In contrast, the TLR2 homodimer mediates beneficial effect of Lactobacillus rhamnosus GG. ${ }^{31}$ Thus, TLR2 homodimer and heterodimer may have distinct functions in obesity and NASH.

\section{TLR4}

TLR4 senses the components of Gram-negative bacteria, including LPS. In addition, TLR4 has been reported to recognize free fatty acids. In this section, we mainly focus on the role of gut-derived LPS. Similar to TLR2, TLR4 signaling leads to the production of proinflammatory cytokines, including $\mathrm{TNF} \alpha, \mathrm{IL}-1$, and IL- 6 . As expected, TLR4 deficient mice are protected from diet-induced obesity by a decrease in the levels of these proinflammatory cytokines.

The total number of Gram-negative bacteria in the gut is not always increased in obesity. However, the plasma LPS levels are elevated in mice on a HF diet. ${ }^{34}$ In addition, the circulating LPS levels are elevated in patients with metabolic syndrome and NAFLD. ${ }^{35}$ Increased gut permeability accounts for the elevation of the plasma LPS levels due to the impaired barrier function. ${ }^{34}$ In mice on a HF diet, the expression of tight junction proteins, including zonula occludens-1 (ZO-1) and occludin, were decreased in the small intestine. ${ }^{10}$ As a result, the gut leaks bacterial components, which stimulate TLR expressing cells. In fact, the sterilization of the gut microbiota restored the tight junction proteins, which subsequently normalized the plasma LPS levels. ${ }^{10}$ Probiotics treatment can maintain the tight junction proteins in mouse models of obesity. ${ }^{36}$ These data suggest that normal gut flora regulates the permeability and prevents the leaks of bacterial components, which can promote the development of obesity.

As a mechanism of the enhanced TLR4 signaling under obese conditions, the expression of TLR4 is increased in the muscle ${ }^{37}$, the visceral fat tissue ${ }^{38}$, and the liver ${ }^{39}$. Monocytes/macrophages contribute to the expression of TLR4 in these organs. ${ }^{40}$ Macrophages are roughly divided into two phenotypes: M1 macrophages, which show a proinflammatory phenotype, while M2 macrophages exhibit an anti-inflammatory phenotype. Most of reports have demonstrated that M1-type macrophages highly express TLR4 and infiltrate the target organs of obesity. On the other hand, M2-type macrophages, which show a lower expression of TLR4, are dominant in lean subjects. The M2 phenotype of AT macrophages is maintained in TLR4 mutant mice even when they were fed a HF diet. ${ }^{41}$ In addition, the M2 phenotype of hepatic macrophage Kupffer cells is maintained in TLR4 mutant mice, while WT mice with NASH show the M1 phenotype. Sohn et al..$^{42}$ demonstrated that the administration of Lactobacillus paracasei promoted the 
shifting of macrophages from the M1 to the M2 phenotype and the subsequent improvement of steatohepatitis. These data demonstrate that the macrophage phenotype is closely associated with the development of obesity and can be revered in response to the surrounding environment. At the intracellular level, TLR4 signaling is activated through the low expression of microRNAs ${ }^{43}$ and negative regulators. ${ }^{44}$ For instance, the levels of microRNA-181a, which can inhibit TLR4 signaling, were lower in monocytes isolated from subjects with metabolic syndrome. ${ }^{43}$ In contrast, the expression of the microRNA was restored by body weight loss. The expression of activating transcription factor 3 , a negative regulator of TLR4 signaling, was decreased under obese conditions. ${ }^{44}$ Thus, TLR4 signaling is accelerated under obese conditions.

TLR4 is associated with AT fibrosis, a recently emerged concept that is involved in the development of metabolic syndrome. AT fibrosis restricts the expansion of adipocytes, leading to adipocyte dysfunction as well as ectopic fat accumulation. Vila et al. ${ }^{45}$ showed that there was a close association between metabolic syndrome and AT fibrosis in patients with obesity. They also investigated the detailed mechanisms using TLR4 mutant mice. A HF diet induced AT fibrosis in WT mice, while TLR4 mutant mice developed a lower grade of fibrosis. As a result, TLR4 mutant mice were protected from obesity and insulin resistance. In addition, a TLR4 inhibitor prevented AT fibrosis while the administration of TLR4 ligand LPS promoted fibrosis by producing transforming growth factor- $\beta 1$ (TGF- $\beta 1$ ), a potent fibrogenic factor. These data revealed that LPS promotes obesity through AT fibrosis. In this model, immune cells including macrophages produced fibrogenic factors through TLR4. Interestingly, the infiltrating macrophages that promoted AT fibrosis showed an M2 or M2-like phenotype, which exert anti-inflammatory effects. This finding is in contrast to most previous reports, which suggested that M1-type macrophages were recruited into the AT when mice were fed a HF diet. Vila et al. ${ }^{45}$ used $\mathrm{C} 3 \mathrm{H}$ background mice, which are prone to AT fibrosis. In $\mathrm{C} 3 \mathrm{H}$ mice, a HF diet induced fibrosis in the AT but not in the liver, suggesting that AT and hepatic macrophages may have different responses to a HF diet. In the commonly used C57BL6 background mice, a weak infiltration of macrophage was observed in the AT and the development of AT fibrosis occurred as a late-onset event with a moderate elevation of fibrogenic factors. It is well known that macrophages can secrete both fibrogenic factors ${ }^{46}$ and anti-fibrogenic factors. ${ }^{47}$ Although TLR4 signaling is associated with the macrophage phenotype, further investigations are necessary to better understand how TLR4 affects the macrophage phenotypes.

Although the effects of TLR4 on immune cells has been extensively investigated, Jia et al. ${ }^{48}$ focused on the TLR4 signaling in hepatocytes and found that hepatocyte-specific TLR4 deficient mice were protected from obesity and insulin resistance. Until this report was published, the role of TLR4 signaling in hepatocytes was underestimated. Hepatocyte-specific TLR4 deficient mice on a HF diet exhibited an interesting phenotype: inflammatory cell infiltration was suppressed in both the liver and the AT, even though the mice exhibit comparable levels of obesity to WT mice. As a result, the expression of inflammatory genes was decreased in hepatocytespecific TLR4 deficient mice. These data suggest that hepatocytes produce chemokines that recruit inflammatory cells in the liver and AT through TLR4 signaling. They also demonstrated that macrophage-specific TLR4 deficient mice were not protected from obesity or the insulin resistance induced by a HF diet. The phenotype of macrophage-specific TLR4 deficient mice was in sharp contrast to previous reports, which stated that the expression of TLR4 on macrophages contributes to the development of inflammation. These data raise the question as to whether the expression of TLR4 on macrophages contributes to inflammation or anti-inflammation. In macrophage-specific TLR4 deficient mice, compensated cytokine production from other immune cells elevated proinflammatory cytokine levels. These data demonstrate that the expression of TLR4 on macrophages also contributes to inflammation by regulating other cells. Thus, TLR4 on macrophages is crucial to triggering inflammation.

\section{MyD88 and TRIF}

TLR4 is the only TLR that uses both MyD88 and TRIF, as adaptor proteins. The other TLRs use either MyD88 or TRIF (Fig. 1). These adaptor proteins are also molecular targets of obesity. MyD88 is well characterized as a classical inflammatory cascade that induces various proinflammatory cytokines, including $\mathrm{TNF} \alpha$, IL-1, and IL-6. MyD88 deficient mice are protected from atherosclerosis in ApoE deficient mice ${ }^{49}$ and in CDAA-induced steatohepatitis and obesity. ${ }^{22}$ On the other hand, MyD88 deficient mice 
showed an increased risk of diabetes even though the levels of $\mathrm{TNF} \alpha$, a key player to induce insulin resistance, were decreased. ${ }^{50}$ Because MyD88 is ubiquitously expressed in many cell types, the role of MyD88 may differ among cells. For instance, MyD88 deletion in the central nervous system ${ }^{51}$ and intestinal epithelial cell $s^{52}$ was protected from HF diet-induced obesity. In contrast, hepatocyte-specific MyD88 depletion resulted in an impaired glucose metabolism. ${ }^{53}$ Since IL-1 and IL-18 also use MyD88 signaling, these cytokines may participate in the development of obesity. Indeed, we have reported that IL-1 receptor deficient mice were protected from steatohepatitis. ${ }^{22}$

As described previously, hepatocyte-specific TLR4 deficient mice but not hepatocyte-specific MyD88 deficient mice were protected from the insulin resistance induced by a HF diet, suggesting the possibility that the TLR4-TRIF pathway in hepatocytes promotes insulin resistance. In line with former reports, TRIF deficient mice were protected from the insulin resistance induced by lard feeding. ${ }^{54}$ However the mice showed an opposite phenotype when they were fed a CDAA diet..$^{55}$ To clarify the role of TRIF at the cell level, we isolated hepatocytes, Kupffer cells, and hepatic stellate cell from the WT and TRIF deficient liver and examined chemokine expression in response to LPS. The response to LPS varies among cells..$^{55}$ Collectively, the roles of MyD88 and TRIF are complex and the function of these adaptor proteins depend on the organ or cells.

\section{TLR5 and TLR9}

TLR5 recognizes bacterial flagellin and transmits the signal through MyD88. The intestinal mucosa expresses TLR5 and contributes to the host defense in the gut. The first report on TLR5 deficient mice had a strong impact on the development of obesity and insulin resistance, in which the composition of the gut microbiota was dramatically altered.$^{56}$ However, TLR5 deficient mice in another animal facility, which were referred to as TLR5KO-2 mice, did not reproduce similar phenotypes to the initially reported TLR5 deficient mice, which were referred to as TLR5KO-1 mice. ${ }^{57}$ In addition, the composition of the gut microbiota was similar between WT and TLR5KO-2 mice but largely different between TLR5KO- 1 and TLR5KO-2 mice. These data indicate that environmental factors are involved in the activation of TLR5.
TLR9 senses viral and bacterial DNA and transmits the signal through MyD88. In addition, DNAs from the injured nucleus and mitochondria can activate TLR9. We have reported that bacterial DNA could be detected in CDAA-fed mice and TLR9 deficient mice were protected from CDAA diet-induced obesity and steatohepatitis ${ }^{22}$, indicating that TLR9 also participates in the development of obesity and NASH. Besides bacterial DNA, nucleic acids can promote TLR9 signaling. Adipocytes and hepatocytes release micro-particles that contain nucleic acids, including microRNA and mitochondrial DNA, under excessive stress. The micro-particles released from adipocytes were increased in mice on a HF diet whereas the clearance of micro-particles was reduced. ${ }^{58}$ As a result, micro-particles containing TLR9 ligands stimulate TLR9 in the immune cells and induce inflammation in the AT. Garcia-Martinez et al. ${ }^{59}$ reported that mitochondrial DNA released from hepatocytes serves as a TLR9 ligand and promotes steatohepatitis. They demonstrated that the mitochondrial DNA levels were elevated in obese patients with abnormal liver function tests. Although TLR9 signaling could induce the anti-inflammatory genes related to interferon regulatory factor 7-dependent pathway, mitochondrial DNA stimulated the inflammatory pathway but not the anti-inflammatory pathway. They also demonstrated that a TLR7/9 antagonist was effective for improving NASH. These data indicate that TLR9 signaling is also a target of treatments for obesity and NASH.

\section{CONCLUSION}

Recently, the role of the gut microbiota has received a great deal of attention in the development of obesity and its related diseases. However, we should pay attention to environmental factors in mice as well as humans. In animal facilities, gene-modified mice were often bred separately for long periods of time, which might have led to the formation of a distinct gut microbiota composition. ${ }^{60} \mathrm{In}$ addition, there are often marked differences in the environmental factors of different countries and the differences can even be observed within the same country. However, it is a fact that the gut microbiota and host-derived factors contribute to the development of obesity and NAFLD as TLR ligands. The activation of TLR signaling induces proinflammatory cytokines, which damage the host beyond the defense against invading pathogens. Currently, probiotics 
and several TLR antagonists have shown favorable results in the treatment of obesity and its related diseases including NAFLD. Thus, the gut microbiota and TLR signaling represent a molecular target for treatment to improve obesity and NAFLD.

\section{CONFLICTS OF INTEREST}

The authors declare no conflict of interest.

\section{ACKNOWLEDGMENTS}

This work was supported by KAKENHI (16K09337).

\section{REFERENCES}

1. World Helath Organization. Fact sheet. Obesity and overwight: Updated June 2016. [accessed 2017 March 2]; Available from: URL: http://www.who.int/mediacentre/factsheets/fs311/en/

2. Kawai T, Akira S. Toll-like receptors and their crosstalk with other innate receptors in infection and immunity. Immunity 2011;34:637-50.

3. Shi H, Kokoeva MV, Inouye K, Tzameli I, Yin H, Flier JS. TLR4 links innate immunity and fatty acid-induced insulin resistance. J Clin Invest 2006;116:3015-25.

4. Miura K, Ohnishi H. Role of gut microbiota and Toll-like receptors in nonalcoholic fatty liver disease. World J Gastroenterol 2014;20:7381-91.

5. Ley RE, Bäckhed F, Turnbaugh P, Lozupone CA, Knight RD, Gordon JI. Obesity alters gut microbial ecology. Proc Natl Acad Sci U S A 2005;102:11070-5.

6. Turnbaugh PJ, Hamady M, Yatsunenko T, Cantarel BL, Duncan A, Ley RE, et al. A core gut microbiome in obese and lean twins. Nature 2009;457:480-4.

7. Hildebrandt MA, Hoffmann C, Sherrill-Mix SA, Keilbaugh SA, Hamady M, Chen YY, et al. High-fat diet determines the composition of the murine gut microbiome independently of obesity. Gastroenterology 2009;137:1716-24.

8. Ley RE, Turnbaugh PJ, Klein S, Gordon JI. Microbial ecology: human gut microbes associated with obesity. Nature 2006;444: 1022-3.
9. Ishioka M, Miura K, Minami S, Shimura Y, Ohnishi H. Altered gut microbiota composition and immune response in experimental steatohepatitis mouse models. Dig Dis Sci 2017;62: 396-406.

10. Cani PD, Bibiloni R, Knauf C, Waget A, Neyrinck AM, Delzenne NM, et al. Changes in gut microbiota control metabolic endotoxemia-induced inflammation in high-fat diet-induced obesity and diabetes in mice. Diabetes 2008;57:1470-81.

11. Hwang I, Park YJ, Kim YR, Kim YN, Ka S, Lee HY, et al. Alteration of gut microbiota by vancomycin and bacitracin improves insulin resistance via glucagon-like peptide 1 in diet-induced obesity. FASEB J 2015;29:2397-411.

12. Vrieze A, Out C, Fuentes S, Jonker L, Reuling I, Kootte RS, et al. Impact of oral vancomycin on gut microbiota, bile acid metabolism, and insulin sensitivity. J Hepatol 2014;60:824-31.

13. Bäckhed F, Ding H, Wang T, Hooper LV, Koh GY, Nagy A, et al. The gut microbiota as an environmental factor that regulates fat storage. Proc Natl Acad Sci U S A 2004;101:15718-23. 14. Jung MJ, Lee J, Shin NR, Kim MS, Hyun DW, Yun JH, et al. Chronic repression of mTOR complex 2 induces changes in the gut microbiota of diet-induced obese mice. Sci Rep 2016; 6:30887.

15. Lee HY, Park JH, Seok SH, Baek MW, Kim DJ, Lee KE, et al. Human originated bacteria, Lactobacillus rhamnosus PL60, produce conjugated linoleic acid and show anti-obesity effects in diet-induced obese mice. Biochim Biophys Acta 2006;1761: 736-44.

16. Ma X, Hua J, Li Z. Probiotics improve high fat diet-induced hepatic steatosis and insulin resistance by increasing hepatic NKT cells. J Hepatol 2008;49:821-30.

17. Alisi A, Bedogni G, Baviera G, Giorgio V, Porro E, Paris C, et al. Randomised clinical trial: the beneficial effects of VSL\#3 in obese children with non-alcoholic steatohepatitis. Aliment Pharmacol Ther 2014;39:1276-85.

18. Everard A, Belzer C, Geurts L, Ouwerkerk JP, Druart C, Bindels LB, et al. Cross-talk between Akkermansia muciniphila and intestinal epithelium controls diet-induced obesity. Proc Natl Acad Sci U S A 2013;110:9066-71.

19. Shin NR, Lee JC, Lee HY, Kim MS, Whon TW, Lee MS, et al. An increase in the Akkermansia spp. population induced by 
metformin treatment improves glucose homeostasis in diet-induced obese mice. Gut 2014;63:727-35.

20. Okubo H, Sakoda H, Kushiyama A, Fujishiro M, Nakatsu Y, Fukushima T, et al. Lactobacillus casei strain Shirota protects against nonalcoholic steatohepatitis development in a rodent model. Am J Physiol Gastrointest Liver Physiol 2013;305: G911-8.

21. Velayudham A, Dolganiuc A, Ellis M, Petrasek J, Kodys K, Mandrekar $P$, et al. VSL\#3 probiotic treatment attenuates fibrosis without changes in steatohepatitis in a diet-induced nonalcoholic steatohepatitis model in mice. Hepatology 2009; 49:989-97.

22. Miura K, Kodama Y, Inokuchi S, Schnabl B, Aoyama T, Ohnishi $\mathrm{H}$, et al. Toll-like receptor 9 promotes steatohepatitis by induction of interleukin-1beta in mice. Gastroenterology 2010; 139:323-34.

23. Miura K, Yang L, van Rooijen N, Brenner DA, Ohnishi H, Seki E. Toll-like receptor 2 and palmitic acid cooperatively contribute to the development of nonalcoholic steatohepatitis through inflammasome activation in mice. Hepatology 2013; 57:577-89.

24. Matsumoto M, Hada N, Sakamaki Y, Uno A, Shiga T, Tanaka C, et al. An improved mouse model that rapidly develops fibrosis in non-alcoholic steatohepatitis. Int J Exp Pathol 2013;94:93-103.

25. Turnbaugh PJ, Ley RE, Mahowald MA, Magrini V, Mardis ER, Gordon JI. An obesity-associated gut microbiome with increased capacity for energy harvest. Nature 2006;444:1027-31.

26. Brenner C, Simmonds RE, Wood S, Rose V, Feldmann M, Turner J. TLR signalling and adapter utilization in primary human in vitro differentiated adipocytes. Scand J Immunol 2012; 76:359-70.

27.Jaedicke KM, Roythorne A, Padget K, Todryk S, Preshaw PM, Taylor JJ. Leptin up-regulates TLR2 in human monocytes. J Leukoc Biol 2013;93:561-71.

28. Ehses JA, Meier DT, Wueest S, Rytka J, Boller S, Wielinga PY, et al. Toll-like receptor 2-deficient mice are protected from insulin resistance and beta cell dysfunction induced by a high-fat diet. Diabetologia 2010;53:1795-806.

29. Rivera CA, Gaskin L, Allman M, Pang J, Brady K, Adegboyega $\mathrm{P}$, et al. Toll-like receptor-2 deficiency enhances non-alcoholic steatohepatitis. BMC Gastroenterol 2010;10:52.

30. Paik YH, Lee KS, Lee HJ, Yang KM, Lee SJ, Lee DK, et al. Hepatic stellate cells primed with cytokines upregulate inflammation in response to peptidoglycan or lipoteichoic acid. Lab Invest 2006;86:676-86.

31. Zhang Z, Zhou Z, Li Y, Zhou L, Ding Q, Xu L. Isolated exopolysaccharides from Lactobacillus rhamnosus GG alleviated adipogenesis mediated by TLR2 in mice. Sci Rep 2016;6: 36083.

32. Arias-Loste MT, Iruzubieta P, Puente Á, Ramos D, Santa Cruz C, Estébanez Á, et al. Increased expression profile and functionality of TLR6 in peripheral blood mononuclear cells and hepatocytes of morbidly obese patients with non-alcoholic fatty liver disease. Int J Mol Sci 2016;17:1878.

33. Moreno-Viedma V, Amor M, Sarabi A, Bilban M, Staffler G, Zeyda $\mathrm{M}$, et al. Common dysregulated pathways in obese adipose tissue and atherosclerosis. Cardiovasc Diabetol 2016;15: 120.

34. Cani PD, Amar J, Iglesias MA, Poggi M, Knauf C, Bastelica D, et al. Metabolic endotoxemia initiates obesity and insulin resistance. Diabetes 2007;56:1761-72.

35. Creely SJ, McTernan PG, Kusminski CM, Fisher fM, Da Silva NF, Khanolkar M, et al. Lipopolysaccharide activates an innate immune system response in human adipose tissue in obesity and type 2 diabetes. Am J Physiol Endocrinol Metab 2007; 292:740-7.

36. Cani PD, Possemiers S, Van de Wiele T, Guiot Y, Everard A, Rottier $\mathrm{O}$, et al. Changes in gut microbiota control inflammation in obese mice through a mechanism involving GLP-2-driven improvement of gut permeability. Gut 2009;58:1091-103.

37. Reyna SM, Ghosh S, Tantiwong P, Meka CS, Eagan P, Jenkinson CP, et al. Elevated toll-like receptor 4 expression and signaling in muscle from insulin-resistant subjects. Diabetes 2008; 57:2595-602.

38. Poulain-Godefroy O, Le Bacquer O, Plancq P, Lecoeur C, Pattou F, Frühbeck G, et al. Inflammatory role of toll-like receptors in human and murine adipose tissue. Mediators Inflamm 2010;2010:823486.

39. Rivera CA, Gaskin L, Singer G, Houghton J, Allman M. Western diet enhances hepatic inflammation in mice exposed to ce- 
cal ligation and puncture. BMC Physiol 2010;10:20.

40. Ahmad R, Al-Mass A, Atizado V, Al-Hubail A, Al-Ghimlas F, Al-Arouj M, et al. Elevated expression of the toll like receptors 2 and 4 in obese individuals: its significance for obesity-induced inflammation. J Inflamm (Lond) 2012;9:48.

41. Suganami T, Mieda T, Itoh M, Shimoda Y, Kamei Y, Ogawa Y. Attenuation of obesity-induced adipose tissue inflammation in $\mathrm{C} 3 \mathrm{H} / \mathrm{HeJ}$ mice carrying a Toll-like receptor 4 mutation. Biochem Biophys Res Commun 2007;354:45-9.

42. Sohn W, Jun DW, Lee KN, Lee HL, Lee OY, Choi HS, et al. Lactobacillus paracasei induces M2-dominant Kupffer cell polarization in a mouse model of nonalcoholic steatohepatitis. Dig Dis Sci 2015;60:3340-50.

43. Hulsmans M, Sinnaeve P, Van der Schueren B, Mathieu C, Janssens S, Holvoet P. Decreased miR-181a expression in monocytes of obese patients is associated with the occurrence of metabolic syndrome and coronary artery disease. J Clin Endocrinol Metab 2012;97:1213-8.

44. Suganami T, Yuan X, Shimoda Y, Uchio-Yamada K, Nakagawa N, Shirakawa I, et al. Activating transcription factor 3 constitutes a negative feedback mechanism that attenuates saturated fatty acid/Toll-like receptor 4 signaling and macrophage activation in obese adipose tissue. Circ Res 2009;105:25-32.

45. Vila IK, Badin PM, Marques MA, Monbrun L, Lefort C, Mir L, et al. Immune cell Toll-like receptor 4 mediates the development of obesity- and endotoxemia-associated adipose tissue fibrosis. Cell Rep 2014;7:1116-29.

46. Keophiphath M, Achard V, Henegar C, Rouault C, Clément K, Lacasa D. Macrophage-secreted factors promote a profibrotic phenotype in human preadipocytes. Mol Endocrinol 2009;23: 11-24.

47. Ramachandran P, Pellicoro A, Vernon MA, Boulter L, Aucott RL, Ali A, et al. Differential Ly-6C expression identifies the recruited macrophage phenotype, which orchestrates the regression of murine liver fibrosis. Proc Natl Acad Sci U S A 2012; 109:E3186-95.

48. Jia L, Vianna CR, Fukuda M, Berglund ED, Liu C, Tao C, et al. Hepatocyte Toll-like receptor 4 regulates obesity-induced inflammation and insulin resistance. Nat Commun 2014;5:3878. 49. Björkbacka H, Kunjathoor VV, Moore KJ, Koehn S, Ordija
CM, Lee MA, et al. Reduced atherosclerosis in MyD88-null mice links elevated serum cholesterol levels to activation of innate immunity signaling pathways. Nat Med 2004;10:416-21.

50. Hosoi T, Yokoyama S, Matsuo S, Akira S, Ozawa K. Myeloid differentiation factor 88 (MyD88)-deficiency increases risk of diabetes in mice. PLoS One 2010;5:e12537.

51. Kleinridders A, Schenten D, Könner AC, Belgardt BF, Mauer J, Okamura T, et al. MyD88 signaling in the CNS is required for development of fatty acid-induced leptin resistance and dietinduced obesity. Cell Metab 2009;10:249-59.

52. Everard A, Geurts L, Caesar R, Van Hul M, Matamoros S, Duparc T, et al. Intestinal epithelial MyD88 is a sensor switching host metabolism towards obesity according to nutritional status. Nat Commun 2014;5:5648.

53. Duparc T, Plovier H, Marrachelli VG, Van Hul M, Essaghir A, Ståhlman M, et al. Hepatocyte MyD88 affects bile acids, gut microbiota and metabolome contributing to regulate glucose and lipid metabolism. Gut 2017;66:620-32.

54. Caesar R, Tremaroli V, Kovatcheva-Datchary P, Cani PD, Bäckhed F. Crosstalk between gut microbiota and dietary lipids aggravates WAT inflammation through TLR signaling. Cell Metab 2015;22:658-68.

55. Yang L, Miura K, Zhang B, Matsushita H, Yang YM, Liang S, et al. TRIF differentially regulates hepatic steatosis and inflammation/fibrosis in mice. Cell Mol Gastroenterol Hepatol 2017; 3:469-83.

56. Vijay-Kumar M, Aitken JD, Carvalho FA, Cullender TC, Mwangi S, Srinivasan S, et al. Metabolic syndrome and altered gut microbiota in mice lacking Toll-like receptor 5. Science 2010;328:228-31.

57.Zhang W, Hartmann R, Tun HM, Elson CO, Khafipour E, Garvey WT. Deletion of the Toll-like receptor 5 gene per se does not determine the gut microbiome profile that induces metabolic syndrome: environment trumps genotype. PLoS One 2016;11:e0150943.

58. Revelo XS, Ghazarian M, Chng MH, Luck H, Kim JH, Zeng K, et al. Nucleic acid-targeting pathways promote inflammation in obesity-related insulin resistance. Cell Rep 2016;16:717-30.

59. Garcia-Martinez I, Santoro N, Chen Y, Hoque R, Ouyang X, Caprio S, et al. Hepatocyte mitochondrial DNA drives nonal- 
coholic steatohepatitis by activation of TLR9. J Clin Invest 2016;126:859-64.

60. Ubeda C, Lipuma L, Gobourne A, Viale A, Leiner I, Equinda
$M$, et al. Familial transmission rather than defective innate immunity shapes the distinct intestinal microbiota of TLR-deficient mice. J Exp Med 2012;209:1445-56. 